\title{
Zwischen Zwangsbeitrag und Demokratieabgabe. Institutionelle Reform der Rundfunkpolitik trotz Politikverflechtung*
}

\section{Kurzfassung}

Rundfunkpolitik hat im deutschen politischen System eine Sonderstellung, die vor allem durch die Rundfunkkommission und die Kommission zur Ermittlung des Finanzbedarfs (KEF) zum Ausdruck kommt. Diese besondere institutionelle Struktur sorgt für eine Staats- und Marktferne der Inhalte und der Finanzierung des Rundfunks. Obwohl die Besonderheiten der Rundfunkpolitik diese anfällig machen für inkrementelle Anpassungen, konnte eine institutionelle Reform erreicht werden. Um das Verteilungsproblem von sinkenden Einnahmen zu lösen, wurde die bisherige Rundfunkgebühr zum 01. Januar 2013 in einen Rundfunkbeitrag umgewandelt. Im Gegensatz zur früheren Gebühr ist seitdem jeder Haushalt und jede Betriebsstätte gezwungen, den Rundfunkbeitrag zu zahlen. Die neue Regelung basiert auf den Ergebnissen des Verhandlungsprozesses zum Rundfunkbeitragsstaatsvertrag. Trotz der horizontalen Politikverflechtung und einem funktionalen Zwangsverhandlungssystem führten ein „Schleier des Nichtwissens“, eine segmentierte Entscheidungsfindung und ein Zusammenwirken von positiver und negativer Koordination zur institutionellen Reform der Rundfunkpolitik.

* Dank gebührt meinen Interviewpartnern Markus Kurze, Sebastian Rehse, Stefan Gebhardt, Claus Peter Boßmann und Hermann Eicher. Weiterhin danke ich Arthur Benz und den Teilnehmern des Seminars „Varianten und Dynamiken der Politikverflechtung“ an der TU Darmstadt, den beiden Gutachtern und der Redaktion der Zeitschrift für Politikwissenschaft. Verbliebene Unzulänglichkeiten liegen in meiner Verantwortung. 


\section{Inhalt}

1. Einleitung 242

a) Die Politikverflechtungstheorie als analytischer Rahmen 243

b) Stand der Forschung 245

2. Institutionelle Ausgangslage 246

a) Rundfunkkommission als funktionales Zwangsverhandlungssystem 246

b) Kommission zur Ermittlung des Finanzbedarfs (KEF) 247

3. Niveau- und Verteilungsproblem der Rundfunkpolitik in Deutschland 249

a) Niveauproblem durch sinkende Akzeptanz des öffentlichen Rundfunks 249

b) Verteilungsproblem 251

4. Inkrementelle Anpassungen als Reaktion auf die Probleme 252

a) Technische Konvergenz 252

b) Finanzierungsmodelle 253

5. Von der inkrementellen Anpassung zur institutionellen Reform - oder von der Gebühr zum Beitrag 255

6. Institutionelle Reform trotz Politikverflechtung 257

a) „Schleier des Nichtwissens“ 258

b) Segmentierte Entscheidungsfindung 259

c) Positive und negative Koordination 260

7. Zusammenfassung und Ausblick 260

\section{Einleitung}

Der Rundfunkbeitragsstaatsvertrag stellt die Finanzierung der öffentlich-rechtlichen Rundfunkanstalten auf eine grundsätzlich neue Basis. Anknüpfungspunkt für den Beitrag ist nicht mehr das einzelne Empfangsgerät, sondern der jeweilige Haushalt oder die Betriebsstätte. Mit anderen Worten: Ab dem 01. Januar 2013 muss jeder den Rundfunkbeitrag leisten. Vorher konnte man noch durch den Verzicht auf Fernseh- und Radiogeräte der Gebühr ,entkommen“. Bezüglich des neuen Beitrags kann nicht mehr argumentiert werden, jemand hätte keinen Haushalt oder keine Betriebsstätte.

Die Meinungen über diesen neuen Rundfunkbeitrag gehen weit auseinander. Einerseits beschwören die öffentlich-rechtlichen Rundfunkanstalten ihn als „Demokratie-Abgabe" (Schönenborn 2012). Andererseits kritisieren den Rundfunkbeitrag vor allem Unternehmen als „Zwangsabgabe“ (Hauser 2013). Das Ergebnis der un- 
terschiedlichen Einschätzungen sind zahlreiche Klagen vor deutschen Gerichten, mit derzeit noch offenem Ausgang (Hanfeld 2013).

Davon unabhängig gelang es den zuständigen Ministerpräsidenten der Länder, die Rundfunkfinanzierung grundsätzlich zu reformieren. Doch aus welchen Gründen konnte diese institutionelle Reform der Rundfunkfinanzierung überhaupt gelingen? Weshalb wurde der Pfad inkrementeller Anpassungen, wie sie in der horizontalen Politikverflechtung auch in der Rundfunkpolitik bisher verbreitet waren, verlassen und eine institutionelle Reform durchgeführt?

Die Rundfunkkommission und die Kommission zur Ermittlung des Finanzbedarfs (KEF) sind der institutionelle Ausgangspunkt der Rundfunkpolitik. Die Probleme der Rundfunkpolitik bestehen aus der Sichtweise der Theorie der Politikverflechtung in einem Niveau- und einem Verteilungsproblem. Im Sinne der Prognosen der Politikverflechtungstheorie zeigen sich in der Rundfunkpolitik lediglich inkrementelle Anpassungen. Diese Anpassungen gehen allerdings mit der Änderung der Gebühr in den neuen Beitrag in eine institutionelle Reform über. Im Ergebnis konnte trotz der horizontalen Politikverflechtung in der Rundfunkpolitik eine institutionelle Reform erreicht werden. Die empirische Frage nach den Gründen für die institutionelle Reform - von der Gebühr zum Beitrag - verbindet sich untrennbar mit der theoretischen Frage nach den Gründen für die Umgehung der Politikverflechtungsfalle.

Dafür waren drei Faktoren des Verhandlungsprozesses verantwortlich: Zum einen entschieden die Akteure unter einem „Schleier des Nichtwissens“. Zum anderen fand eine segmentierte Entscheidung statt, wodurch der Verhandlungsprozess die Teilpolitik „Einnahmen der Rundfunkfinanzierung“ isolierte. Andere Teilpolitiken und auch Finanzierungsmodelle wurden dadurch schrittweise vom Verhandlungsprozess ausgeschlossen. Schließlich war das Zusammenwirken positiver und negativer Koordination für die institutionelle Reform ausschlaggebend. So wurde zwar das Verteilungsproblem gelöst und der Rundfunk mit ausreichend finanziellen Mitteln für die Zeit zwischen 2013 und 2016 ausgestattet, aber das Niveau des Rundfunks im Sinne seiner Akzeptanz ist weiter gesunken.

\section{a) Die Politikverflechtungstheorie als analytischer Rahmen}

Nach dem Zweiten Weltkrieg zogen die Alliierten Lehren aus der Medienpolitik und Propaganda des Nationalsozialismus und dezentralisierten die Rundfunkpolitik in Deutschland. Damit zählt die Rundfunkpolitik zur horizontalen Politikverflechtung, weil nur die Länder als die dezentralen Entscheidungseinheiten beteiligt sind (Scharpf/Reissert/Schnabel 1976: 34). Scharpf u. a. definierten Politikverflechtung 
„als eine der verfügbaren Möglichkeiten, den Steuerungsbedarf dezentralisierter Entscheidungssysteme zu befriedigen und damit spezifische Problemerzeugungstendenzen abzubauen“ (ebd.: 21). Die Entstehung von Problemen im deutschen Bundesstaat begründet die Theorie der Politikverflechtung aus der Sichtweise der ökonomischen Theorie des Föderalismus (Ostrom 1971; Oates 1972): Dezentral ausgeführte Politik lässt Externalitäten entstehen, verbraucht gemeinsame Ressourcen oder erzeugt öffentliche Güter. Doch die drei abstrakten Probleme der ökonomischen Theorie des Föderalismus treten in Deutschland in unterschiedlichen konkreten Typen auf. Für die Theorie der Politikverflechtung sind das Niveau-, Niveaufixierungs-, Verteilungs- und Interaktionsprobleme (Scharpf/Reissert/Schnabel 1976: 25 ff.).

Der Rundfunk ist ein öffentliches Gut (,joint product“) ähnlich anderer Güter, die Anstalten (z. B. Studienplätze), Behörden (z. B. Genehmigungen) oder Unternehmen (z. B. Wasser) im Auftrag der Länder erbringen. Die Kosten für ,joint products" lassen sich auf eine bestimmte Art und Weise verteilen. Für die Theorie sind die ,joint products“ allerdings nicht immer positiv, sondern können unter normativen Gesichtspunkten auch als negativ betrachtet werden (Scharpf 1978: 25). Oft gilt für die Kostenverteilung der Grundsatz ,pay as you use“, so dass der Verbrauch und nicht das Angebot zur Berechnung der Kosten herangezogen wird. Ein Verteilungsproblem entsteht, wenn die Produktionskosten des, joint products“ nicht mehr gedeckt sind. Ein Niveauproblem entsteht, wenn die Konsumenten des öffentlichen Guts die Kostenverteilung der Produzenten nicht mehr als legitim erachten. Für den öffentlichen Rundfunk stellt sich mit Blick auf das Niveauproblem die Frage, ob sich die Kosten auf den Verbrauch oder das Programmangebot beziehen.

Die Theorie der Politikverflechtung besteht aus einem System von insgesamt zwölf Instrumenten, ${ }^{1}$ die Regierungen einsetzen, um den genannten Problemerzeugungstendenzen zu begegnen. Diese verschiedenen Instrumente bewirken eine Konfliktbearbeitung und Konsensbildung. Die Instrumente reduzieren die Zahl der Beteiligten, die Komplexität und die Entscheidungsalternativen. Damit gelingt es den Akteuren zumeist, Niveauprobleme zu lösen, aber die Instrumente eignen sich nicht für eine Reduktion des Problemdrucks bei Verteilungs- oder Interaktionsproblemen (Scharpf 1978: 27). Scharpf u. a. führen dies besonders auf die Instrumente der segmentierten Entscheidungsfindung und der negativen Koordination zurück (Scharpf/Reissert/Schnabel 1976: 66). Positive Koordination bezeichnet eine ge-

1 Dies sind Bilateralisierung, Entflechtung, Fraktionsbildung, Spezialisierung, negative Koordination, Entscheidungs-Segmentierung, Koordination von unten, Strukturerhalt, Gleichbehandlung, Besitzstandswahrung, Konfliktvertragung, Eingriffsverzicht (Scharpf/Reissert/Schnabel 1976: 54 ff.). 
genseitige Abstimmung von allen Teilpolitiken. In der negativen Koordination werden die Folgen von nur einer Teilpolitik für die Akteure diskutiert. Die Einteilung von ganzen Politikbereichen in Segmente und Sequenzen von Teilpolitiken durch die segmentierte Entscheidungsfindung hält die Komplexität gering, so „daß das Verflechtungssystem trotz seiner beschränkten Konsensbildungsfähigkeit auch komplexere Problemzusammenhänge verarbeiten kann“"(ebd.: 61).

Unter Rückgriff auf die Theorie der Politikverflechtung lässt sich für die Rundfunkpolitik keine Lösung von Verteilungsproblemen prognostizieren. Diese Prognose lässt sich mit der Politikverflechtungsfalle und dem funktionalen Zwangsverhandlungssystem in der Rundfunkkommission begründen. Im deutschen Föderalismus können Regierungen keine materiellen Reformen durchführen und auch institutionelle Reformen gelingen nicht. In der Konsequenz verändern sich politische Inhalte im deutschen Föderalismus inkrementell (Scharpf 1985).

Die Unmöglichkeit, Verteilungsentscheidungen in horizontalen Verflechtungssystemen zu treffen, zeigt die Rundfunkpolitik anschaulich. In Deutschland ist die Rundfunkkommission für den Rundfunk verantwortlich; daneben etablierte sich das sogenannte KEF-Verfahren, um die Produktionskosten des öffentlichen Gutes Rundfunk zu ermitteln und zu verteilen.

\section{b) Stand der Forschung}

Die Theorie der Politikverflechtung beschreibt die Besonderheit des deutschen Föderalismus am besten, so dass es in den letzten 40 Jahren Gegenstand zahlreicher Forschungsarbeiten gewesen ist. Gleichzeitig hat Politikverflechtung und der Fokus auf die Politikverflechtungsfalle auch die öffentliche Debatte geprägt (Scheller 2008). Es liegen umfassende Analysen zu Politikfeldern in Deutschland vor, bei denen allerdings die Rundfunkpolitik fehlt (Scheller/Schmid 2008). Erst recht bleibt die Rundfunkpolitik in Überblicksdarstellungen zur Politikverflechtungstheorie (Krick/Blumenthal 2013) und in Lehrbüchern (Kropp 2010) unberücksichtigt. Zwar kennt die Forschung empirische Studien zur Politikverflechtung und der deutschen Wiedervereinigung (Wachendorfer-Schmidt 2003), zum Finanzausgleich (Renzsch 1991) und zur regionalen Wirtschaftsförderung (Nägele 1996), aber bisher blieb die Rundfunkpolitik ein blinder Fleck. Aktuelle Beiträge zum Rundfunkbeitragsstaatsvertrag erkennen zwar die Empirie, erreichen aber keine theoriegleitete Untersuchung dazu (Heinz 2012) oder befassen sich mit juristischen Verfahrensfragen (Knothe 2010).

Die Theorie zeigte sich anpassungsfähig an die von ihr nicht vorhergesagten Entwicklungen der europäischen Integration (Scharpf 2006). Seitdem wurden auch Po- 
litikfelder der EU untersucht (Falkner 2010). Vereinzelt verfolgen Forschungsarbeiten die Frage, ob sich die Politikverflechtung durch die europäische Integration verdoppelt habe (Hrbek 1986; Große-Hüttmann 2004; Moore/Eppler 2008).

Zur horizontalen Politikverflechtung entstanden grundlegende theoretische Erweiterungen (Benz/Scharpf/Zintl 1992), weil die ursprüngliche Theorie der Politikverflechtung sie nur am Rande erwähnt. Erst diese späteren Untersuchungen behandeln Bedingungen, unter denen es in der horizontalen Politikverflechtung zu Entscheidungen kommt. Demnach spielen Koppelgeschäfte, Ausgleichszahlungen und Paketlösungen für Entscheidungen eine zentrale Rolle (Scharpf 1992: 65 ff.). Erst wenn die Ausgleichszahlungen, Koppelgeschäfte und Paketlösungen legitimen Normen, wie etwa Fairness oder Gerechtigkeit, folgen, führen sie zu Entscheidungen (Zintl 1992: 115). Die Verteilung der Kosten der Rundfunkpolitik folgt jedoch keinen Verteilungsnormen, die die Gesellschaft allgemein akzeptiert. ${ }^{2}$ Vor diesem Hintergrund ist die Tatsache, dass es trotz der horizontalen Politikverflechtung zu Entscheidungen in der Rundfunkpolitik kam, erklärungsbedürftig.

\section{Institutionelle Ausgangslage}

\section{a) Rundfunkkommission als funktionales Zwangsverhandlungssystem}

Die Zuständigkeit der Länder für die Rundfunkpolitik sichert Meinungspluralität und beugt damit Machtkonzentration vor (Kops 2009: $6 \mathrm{f}$.). Bis heute verfügt die Rundfunkpolitik über eine besondere Stellung, denn sie ist nicht in einem Fachressort angesiedelt, sondern direkt in den Staats- und Senatskanzleien der Regierungschefs. Rundfunkpolitik ist damit in den Ländern automatisch immer „Chefsache“. In der Koordination zwischen den Ländern ergibt sich eine weitere Besonderheit: Da es für das Politikfeld Rundfunk keine zuständigen Minister gab und gibt, etablierte sich die Rundfunkkommission in der Ministerpräsidentenkonferenz (MPK). Sobald sich die MPK mit Rundfunkpolitik befasst, handeln die Ministerpräsidenten als Rundfunkkommission. Das gleiche gilt für die Chefs der Staats- und Senatskanzleien (CdS) sowie für die Referenten der Arbeitsebene. Während in der MPK der Vorsitz nach einem festgelegten Turnus wechselt, obliegt der Vorsitz der Rundfunkkommission stets dem Bundesland Rheinland-Pfalz bzw. dem dortigen amtierenden Ministerpräsidenten. Damit ist der rheinland-pfälzische Ministerpräsident stets zur Rolle des neutralen Maklers verpflichtet. Enden Staatsverträge oder Finanzierungsperioden, ist es die Aufgabe des Bundeslandes Rheinland-Pfalz, weitere

2 Hieraus speist sich die bereits erwähnte Klagewelle und die oftmals bemühte Bezeichung „Zwangsabgabe" für den Rundfunkbeitrag. 
Prozesse anzustoßen bzw. Ambitionen der Rundfunkkommission und der KEF zu unterstützen. Die Kompetenz über Rundfunkpolitik in einem Land zu bündeln, spricht für die Arbeitsteilung unter den Ländern und sorgt für Kontinuität und Planungssicherheit. Dennoch bleibt die Kompetenz für den Rundfunk geteilt, muss aber von den Ländern gemeinsam ausgeführt werden.

Der Grund für den Zwang zur gemeinsamen Ausführung liegt in der unitarischen politischen Kultur Deutschlands (Jeffery 2008: 589). Diese Besonderheit erfordert einen national einheitlichen Rundfunk und macht Entscheidungen einzelner Bundesländer jenseits des Entscheidungsverbundes unmöglich. Daraus resultiert zwar kein institutionelles Zwangsverhandlungssystem oder „herschaftsförmige“ Politikverflechtung (Kropp 2010: 12), wie es zum Beispiel in den Gemeinschaftsaufgaben der Fall ist. Allerdings ist der faktische Druck auf die Länder, sich zu einigen, so hoch, dass die Rundfunkkommission ein funktionales Zwangsverhandlungssystem darstellt. Der Zwang auf alle Akteure, eine Einigung zu finden, und die Drohung jedes einzelnen Akteurs, eine Einigung zu blockieren, provoziert den Erhalt des Status quo und erschwert institutionelle Reformen. Da alle Bundesländer von demokratiefördernden Diskursen profitieren sind die Länder gezwungen, auch an der markt- und staatsfernen Organisation des Rundfunks mitzuwirken.

Da die Länder diese Zuständigkeit über den Rundfunk dezentral ausüben, muss ein Staatsvertrag zwischen den Ländern geschlossen werden, um einheitliche Regelungen für einen gemeinsamen Rundfunk zu garantieren. Das dazugehörige Verfahren ist sehr anspruchsvoll, weil die Länder nicht nur einen Vertrag zwischen den Regierungen schließen, sondern gleichzeitig ein Gesetz in den jeweiligen Landesparlamenten verabschieden müssen (Knothe 2010).

\section{b) Kommission zur Ermittlung des Finanzbedarfs (KEF)}

Zur dezentralen Organisation des Rundfunks gehört, dass die Inhalte maßgeblich in den Händen der Intendanten liegen. Zwar unterliegen die Intendanten der Kontrolle durch Verwaltungs- und Rundfunkräte, aber sie verfügen trotzdem über eine große Programmautonomie. Dadurch wurde der Rundfunk von gesellschaftlichen Strömungen und politischen Mehrheiten abgekoppelt. ${ }^{3}$ In ähnlicher Weise erfolgte die Finanzierung des öffentlich-rechtlichen Rundfunks unabhängig von der Politik und

3 Die Entkopplung des Rundfunks von der Politik führte zu Klagen vor dem Bundesverfassungsgericht (BVerfG), wenn sich etwa Ministerpräsidenten in die Personalpolitik der Rundfunkanstalten einmischen, wie dies im Fall des ehemaligen ZDF-Chefredaktuers Nikolaus Brender geschah. An diesem Fall zeigt sich, dass Rundfunkanstalten die Staatsferne durch das BVerfG überprüfen lassen und notfalls auch einfordern können. 
unbeeinflusst von wirtschaftlichen Zyklen, so dass der Rundfunk unabhängig von Rezessionen oder ökonomischen Boom-Phasen sicher finanziert werden kann.

Dem Ziel der Neutralität folgend, existiert für die Rundfunkfinanzierung seit dem 20. Februar 1975 das sogenannte KEF-Verfahren. Die KEF prüft die Finanzbedarfe der öffentlich-rechtlichen Rundfunkanstalten und spricht der MPK Empfehlungen über die Höhe des zu entrichtenden Betrags aus. Ein besonderes Augenmerk legt die KEF auf die Frage, ob sich die Anmeldungen der Finanzbedarfe der Rundfunkanstalten im Rahmen des Rundfunkauftrags bewegen und ob sie den Grundsätzen der Wirtschaftlichkeit und Sparsamkeit folgen.

Die KEF besteht aus 16 unabhängigen Sachverständigen, die von den Ministerpräsidenten jeweils für die Dauer von fünf Jahren berufen werden. Aus ihrer Mitte wählt sie einen Vorsitzenden und seinen Stellvertreter. Jedes Land benennt ein Mitglied. Drei Mitglieder kommen aus der Wirtschaftsprüfung und Unternehmensberatung, zwei Mitglieder aus der Betriebswirtschaft. Von ihnen wird Fachkundigkeit in Personal- oder Investitions- und Rationalisierungsfragen verlangt. Zwei Mitglieder verfügen über besondere Erfahrung auf dem Gebiet des Rundfunkrechts und haben die Befähigung zum Richteramt. Drei Mitglieder stammen aus der Medienwirtschaft und der Medienwissenschaft. Ein Mitglied kommt aus dem Bereich der Rundfunktechnik und fünf Mitglieder gehören zu den Landesrechnungshöfen. ${ }^{4}$

Es hat sich in Laufe der Zeit eingebürgert, dass die KEF die Höhe der Finanzbedarfe der öffentlich-rechtlichen Anstalten nach unten korrigiert. Die Finanzbedarfe gelten für eine mehrjährige Periode, damit das KEF-Verfahren regelmäßig die Höhe der Finanzen neu festlegen kann. Genauso wie die Rundfunkkommission hat die KEF ihren ständigen Sitz in Mainz, der Landeshauptstadt von Rheinland-Pfalz, in der das Zweite Deutsche Fernsehen (ZDF) ein Sendezentrum unterhält und der Südwestrundfunk (SWR) ansässig ist.

Die Sonderrolle der Rundfunkpolitik ist auf die hohe Bedeutung des Rundfunks für die Demokratie in Deutschland zurückzuführen. Der Rundfunk bestimmt mit, wie über Politik im Allgemeinen gesprochen wird. Rundfunkpolitik ist wegen ihrer Bedeutung für einen Legitimation stiftenden Diskurs in Demokratien immer auch Verfassungspolitik (Sarcinelli 2006: 200). Im Bund gibt es für die Rundfunkpolitik keinen Gegenpart, so dass Bundestag und Bundesregierung für Probleme in der Rundfunkpolitik nicht zuständig sind. Das Bundesverfassungsgericht wacht darüber, dass der Rundfunk in Deutschland nicht nur fern vom Staat, sondern vor allem fern von der Bundespolitik organisiert wird. Selbst wenn eine Initiative des Bundes für die Bearbeitung von Problemen wünschenswert für die Länder wäre, dürfte sich

4 Genauer www.kef-online.de. 
der Bund aus verfassungsrechtlichen Gründen nicht einmischen. So untersagte das Bundesverfassungsgericht dem Bund 1961 die Errichtung eines bundesweiten Deutschland-Fernsehens, weil die Gesetzgebungskompetenz dafür bei den Ländern läge. ${ }^{5}$ Damit sind die Länder in der Rundfunkpolitik im Positiven wie im Negativen mit Problemen auf sich alleine gestellt.

\section{Niveau- und Verteilungsproblem der Rundfunkpolitik in Deutschland}

\section{a) Niveauproblem durch sinkende Akzeptanz des öffentlichen Rundfunks}

Rundfunk in Deutschland erzeugt Externalitätenprobleme, weil Rundfunkprodukte eines Landes auch in anderen Ländern konsumiert werden können. Auch in der Rundfunkpolitik kann eine, für Externalitätenprobleme charakteristische, Inkongruenz von Verbrauch und Erzeugung festgestellt werden (Scharpf/Reissert/Schnabel 1976: 25). Das Problem verschärfte sich durch die Umstellung der Datenübertragung von Kabelnetzen auf Funknetze. Für Deutschland zeigte sich dies während der Teilung nicht als Problem, sondern als großer Vorteil. Die meisten Menschen in der ehemaligen DDR konnten Westfernsehen und -radio empfangen und dessen Inhalte mit der offiziellen Propaganda der sozialistischen Staatsführung kontrastieren. Auch heute sorgt dieses Externalitätenproblem in autokratischen Staaten für die Verbreitung von demokratischen und freiheitlichen Gedanken, die nur unter großen Anstrengungen autoritärer Regime einzudämmen sind.

Folgt man den Prämissen der Theorie der Politikverflechtung, zeigen sich in der Rundfunkpolitik zwei unterschiedliche Probleme: Für Rundfunkinhalte besteht das schon erwähnte Externalitätenproblem; in der Rundfunkfinanzierung ergibt sich das ,joint product“-Problem. Hierauf folgen unterschiedliche Dezentralisierungsprobleme (Scharpf/Reissert/Schnabel 1976: 23 ff.): das Niveauproblem für die Rundfunkinhalte und das Verteilungsproblem für die Rundfunkfinanzierung.

Die normative Bewertung des Niveaus des öffentlich-rechtlichen Rundfunks im Sinne der Akzeptanz war im Laufe der Zeit diversen Veränderungen ausgesetzt. In der Gründungszeit der Bundesrepublik und nach den Erfahrungen in der Zeit des Nationalsozialismus waren neutrale Rundfunkinhalte ein hohes Gut. Sie standen im Kontrast zur nationalsozialistischen und zur sozialistischen Propaganda und waren nicht immer verfügbar.

Besonders in der Gründungsphase der Bundesrepublik waren Radio und Fernsehen noch wenig verbreitet. Im Jahr 1955 zählte man 100.000 Fernsehgeräte, knapp zehn Jahre später schon sieben Millionen (Hicketier 1998). Heute sind Rundfunk-

5 BVerfGE 12, 205-264 Deutschland-Fernsehen-GmbH (1961). 
inhalte kein Luxusgut mehr, sondern alltäglich und pausenlos verfügbar. Mit einer Vielzahl audiovisueller Geräte lassen sich Radio, Fernsehen und Internet abrufen.

War das Niveau im Sinne einer Akzeptanz von öffentlich-rechtlichen Rundfunkinhalten aus normativen Gründen früher hoch, so kann angenommen werden, dass die Akzeptanz im Laufe der Zeit gesunken ist. Der 18. KEF-Bericht schildert eine deutliche Entwicklung, die eine gesunkene Akzeptanz vermuten lässt. So sank von 2005 bis 2012 die Zahl der angemeldeten Fernsehgeräte um etwa 1,5 Millionen und die Zahl der angemeldeten Hörfunkgeräte um etwa 2 Millionen. ${ }^{6}$ Auch die Sehdauer und die Marktanteile der öffentlich rechtlichen und privaten Fernsehprogramme halten sich mittlerweile die Waage. ${ }^{7}$ Ob aber die Anzahl der angemeldeten Geräte, die Sehdauer und die Marktanteile geeigente Indikatoren für die Akzeptanz des öffentlichen Rundfunks sind, bleibt diskussionswürdig.

Zur postulierten Verminderung des Niveaus von Rundfunkinhalten hat die technische Konvergenz ${ }^{8}$ maßgeblich beigetragen. Demzufolge wachsen Medien, Telekommunikation und IT-Technologie zunehmend zusammen. Technologische Innovationen sorgen dafür, dass Fernseh- und Rundfunkinhalte auch über das Internet transportiert werden können. Gleichzeitig können Fernsehbilder und Radioprogramme nicht nur über Fernseh- und Radionetze, sondern auch über Telefonnetze empfangen werden. Auch wenn die Verteilernetze zusammenwachsen, sich dabei jedoch nicht vollständig substituieren, verschwimmen die Grenzen zwischen Endgeräten, Netzen, IT-Technologie, Fernsehen und Radio zunehmend. Es fallen zwar keine Technologien weg, aber es entstehen neue Anwendungsformen, die es ermöglichen, Rundfunkinhalte zu konsumieren. Schon früher war klar, dass die Speicherchiptechnologie „eher die Vielfalt der Endgeräte [befruchtet]“ (Hoff 2001: 2), statt dass sie Verteilernetze ersetzt. Dies steht in einem starken Gegensatz zur Anfangszeit des öffentlichen Rundfunks, da es damals noch keine tiefe Durchdringung der Gesellschaft mit Radio- bzw. Fernsehgeräten gab.

6 18. KEF-Bericht vom Dezember 2011, S. 171 Abb. 27/1, http://www.kef-online.de/inhalte/bericht18/kef_18bericht.pdf (Stand: 10.9.2013).

7 ARD Jahrbuch 2005, Rundfunk als ,,public value“, Fernsehnutzung 2004, S. 383, http://www.ard.de/ intern/pressearchiv/ard-jahrbuch/-/id=2235058/nid=2235058/did=217804/1rlvwu8/index.html (Stand: 15.11.2013).

8 Die Bezeichnung technische Konvergenz umschreibt das Zusammenwirken mobiler werdender Endgeräte aufgrund der Verschmelzung der Übertragungsnetze. 


\section{b) Verteilungsproblem}

Unter der Bedingung, dass eine Nutzung nur über begrenzt verfügbare Endgeräte möglich war, hatte die Rundfunkgebühr noch den Charakter einer tatsächlichen Gebühr. Entsprechend einfach fiel die Prüfung aus, ob eine Gebührenpflicht bestand oder nicht. Doch mittlerweile ist der Empfang von öffentlichem Rundfunk nicht nur mit Radio- und Fernsehgeräten, sondern zum Beispiel auch mit Handys, Computern, Laptops, Smartphones oder Tablet-Computern möglich. Eine Prüfung auf Gebührenpflicht erweist sich unter diesen Bedingungen als wesentlich komplizierter. Gleichzeitig erscheint die Annahme unplausibel, dass jemand absolut kein rundfunkkompatibles Endgerät benutzt. Bei der generellen Unterstellung, jeder Haushalt und jede Betriebsstätte verfüge über gebührenpflichtige Empfangsgeräte, gibt es zwar eine Minderheit, auf die dies nicht zutrifft. Sie muss jedoch diese ungerechtfertigte Unterstellung tolerieren (Kirchhof 2010: 14; kritisch dazu Degenhart 2011). Wenn nun aber jeder zumindest ein Empfangsgerät zu nutzen scheint, verliert die Gebühr ihren eigentlichen Charakter und entwickelt sich zu einem faktischen Beitrag.

Eine Überprüfung der Gebührenpflicht durch die Gebühreneinzugszentrale (GEZ) und den Beauftragtendienst musste als Gängelung empfunden werden, weil eine Überprüfung nur noch mit einem Eingriff in die Grundrechte (Artikel 13 Grundgesetz, Privatsphäre der Wohnung) erfolgen konnte. Vor allem das Gefühl der Gängelung sorgte für einen großen Akzeptanzverlust des öffentlichen Rundfunks und war ein weiterer Grund für den wachsenden Problemdruck (Reiter 2010: 14). Dies schlug sich auch in der Finanzierung des öffentlich-rechtlichen Rundfunks nieder, weil immer weniger Nutzer ihre Geräte anmeldeten. Damit sind das Niveauund das Verteilungsproblem nicht so scharf voneinander abgrenzbar, wie es den Anschein hat.

Gleichzeitig änderte sich das Konsumverhalten der Fernseh- und Radionutzer. Tendenziell konsumierten sie mehr Inhalte aus privaten Fernseh- und Radiosendern. Der Konsum öffentlich-rechtlicher Inhalte nahm hingegen ab (Meulemann 2009: 7, 10, 11). Dies hatte zur Folge, dass immer mehr Nicht-Nutzer das Personal und die Infrastruktur der öffentlich-rechtlichen Rundfunkanstalten mitfinanzieren mussten. Auch stieg die Rundfunkgebühr in der Vergangenheit kontinuierlich an. Hinsichtlich der Finanzierungsunsicherheit muss konstatiert werden, dass trotz der steigenden Gebühr (Tab. 1) die absoluten Erträge rückläufig waren. 
Tabelle 1: Gesamtbetrag der monatlichen Rundfunkgebühr (Grundgebühr oder Hörfunkgebühr plus Fernsehgebühr)

\begin{tabular}{|l|c|c|c|c|c|c|c|c|c|c|}
\hline Jahr & 1974 & 1979 & 1983 & 1988 & 1990 & 1992 & 1997 & 2001 & 2005 & 2009 \\
\hline Betrag in $€$ & 5,37 & 6,65 & 8,31 & 8,49 & 9,71 & 12,20 & 14,40 & 16,15 & 17,03 & 17,98 \\
\hline
\end{tabular}

Quelle: Kommission zur Ermittlung des Finanzbedarfs, http://www.kef-online.de/inhalte/entwicklung.html (Stand: 22.8.2013).

Die Erträge aus dem Jahr 2012 entsprechen eher dem Gesamtvolumen des Jahres 2008. Im Jahr 2009 hatten die Erträge ein Gesamtvolumen von ca. 7,6 Milliarden $€$. Bei gleichbleibender Gebühr und unveränderter Struktur hätten die Gebühren im Jahr 2016 einen Ertrag von ca. 6,9 Milliarden $€$ eingebracht (Schächter 2010: 9). Die Differenz folgt aus der steigenden Anzahl von Befreiungen und der sinkenden Anzahl von Geräteanmeldungen (Färber/Lücker 2011: 7).

Eine neue Finanzierungsgrundlage musste das Verteilungsproblem aufgrund sinkender Geräteanmeldungen und damit verbundenen sinkenden Einnahmen lösen. Doch Verteilungsprobleme lassen sich in verflochtenen Entscheidungsstrukturen schwer lösen. So zeigen Arbeiten zur Politikverflechtungsfalle, dass verflochtene Entscheidungssysteme bei der Bearbeitung von Problemen weder in materiellen Inhalten noch in institutionellen Grundlagen Problemlösungen erreichen (Scharpf 1985: 329). Die wechselnden Versuche, Inhalte und Institutionen zu ändern, führen demnach zu inkrementellen Änderungen und nicht zu institutionellen Reformen. Übertragen auf die Rundfunkpolitik ließen sich bestenfalls inkrementelle Anpassungen erwarten.

\section{Inkrementelle Anpassungen als Reaktion auf die Probleme}

\section{a) Technische Konvergenz}

Für viele bisherige Entscheidungen in der Rundfunkpolitik treffen Prognosen der Politikverflechtungstheorie zu, weil bestenfalls inkrementelle Anpassungen vorgenommen wurden. Der 12. Rundfunkgebührenstaatsvertrag verfolgte bereits eine inkrementelle Anpassung durch die Unterscheidung zwischen herkömmlichen und neuartigen Empfangsgeräten. Diese Unterscheidung versuchte, Computer als weiteres Endgerät (genauso wie Fernsehen und Radio) juristisch zu etablieren, so dass sich die Anmeldepflicht auch auf diese erstreckt. Seit dem Jahr 1999 umfasste der Rundfunkstaatsvertrag auch das (für den Rundfunkstaatsvertrag) neuartige Internet. Unter den damaligen Bedingungen (Netzqualität, Übertragungsraten, Kostenstruktur, Rechneranforderungen etc.) stellte der Konsum von Radio und Fernsehen über das Internet noch eine seltene Ausnahme dar. Der Rundfunkgebührenstaatsvertrag 
entband in $\S 5$ a zunächst bis Ende des Jahres 2003 Computer von der Gebührenpflicht. Obwohl sich danach der Gesetzestext änderte, hatte dies für die wirkliche Anwendung keine Konsequenzen. Das Gebührenmoratorium für Computer stellte ebenfalls wie die Unterscheidung zwischen herkömmlichen und neuartigen Empfangsgeräten eine inkrementelle Anpassung dar. Das Moratorium wurde zwei Mal bis zum Ende des Jahres 2006 verlängert.

Die technische Entwicklung sorgte während der Dauer des Moratoriums für verbesserte Möglichkeiten, Fernsehen und Radio nun auch auf Computern empfangen zu können. Die MPK reagierte auf diese Entwicklung, indem das Moratorium ab dem 01. Januar 2007 nicht mehr verlängert wurde. Stattdessen war seitdem eine Computer-Gebühr zu entrichten. Sie war in der Folge Gegenstand vieler Gerichtsverfahren, die allerdings lange auf eine klare Antwort zugunsten der ComputerGebühr warten ließen. ${ }^{9}$ Indes endete die technische Entwicklung nicht beim Empfang von Radio und Fernsehen auf dem Personal Computer, sondern sie ermöglichte es auch, auf zahlreichen anderen Geräten Radio und Fernsehen zu empfangen. Angesichts dieser Entwicklung wollten die öffentlich-rechtlichen Rundfunkanstalten und die Rundfunkkommission nicht für jedes neu entwickelte Empfangsgerät eine einzelne Gebührenpflicht feststellen. Die ohnehin hohen Anforderungen an die Kontrolltätigkeit hätte dies weit über die Grenzen des Erträglichen gestreckt, vor allem weil die modernen Empfangsgeräte (Smartphones, Tablet-Computer etc.) mobil und leicht zu verstecken sind. Das Ende des Moratoriums für die Gebühren auf Computer zum Jahresende 2006 veranlasste die MPK in ihrer Sitzung in Bad Pyrmont am 19. und 20. Oktober 2006, die Rundfunkkommission zu beauftragen, neue Finanzierungsmodelle für den öffentlich-rechtlichen Rundfunk zu erarbeiten.

\section{b) Finanzierungsmodelle}

Über ein neues Finanzierungsmodell wollte die Rundfunkkommission nicht nur die genannten Probleme lösen, sondern das Modell musste vor allem unabhängig von Empfangsgeräten, aufkommensneutral und sozial verträglich sein. ${ }^{10}$ Diese Vorgaben stellten die grundsätzlichen Parameter für denkbare Lösungsmodelle dar. $\mathrm{Zu}$ den Bedingungen gehörte auch, dass sich der nicht private Bereich bzw. die Wirtschaft an der Rundfunkfinanzierung beteiligte. Genauso war eine weitere Bedin-

9 Die Rechtmäßigkeit der Computer-Gebühr wurde durch Verwaltungsgerichte sowohl bestätigt als auch verneint und durch das Bundesverwaltungsgericht in drei Fällen letztendlich bestätigt.

10 Institut für Urheber- und Medienrecht, Begründung zum Fünfzehnten Staatsvertrag zur Änderung rundfunkrechtlicher Staatsverträge (Fünfzehnter Rundfunkänderungsstaatsvertrag), http://www.u rheberrecht.org/law/normen/rstv/RStV-15/materialien/00_Begruendung_Allgemeines.php3 (Stand: 20.8.2013). 
gung, dass ein neues Modell sozial begründete Ausnahmen beinhalten musste. Zusätzlich sollten die Gebühr bei 17,98 € konstant bleiben und Gesamteinnahmen von ca. 7,5 Mrd. € erzielt werden.

Daraufhin zirkulierten alternative Modelle zwischen der MPK, den CdS und den Fachreferenten. Gemäß der Aufgabe der MPK entwarfen die Fachreferenten für das regelmäßige Treffen der Ministerpräsidenten vom 17. bis 19. Oktober 2007 unterschiedliche Finanzierungsmodelle und einen Zeitplan für die weiteren Schritte. Dabei griff die Rundfunkkommission auf Modelle zurück, die bereits in den Jahren 1999/2000 entwickelt worden waren. Bereits bevor die Rundfunkkommission ein Moratorium für Computer beschloss, diskutierte sie neue Finanzierungsmodelle. Der Problemdruck erschien allerdings damals noch zu niedrig, um institutionelle Reformen in Angriff zu nehmen. Die CdS konnten weder 1999/2000 noch 2007/2008 eines der vorgeschlagenen Modelle ausschließen, so dass die MPK diese Entscheidung treffen musste. Da von europäischen Institutionen keine Probleme bei der Finanzierung des öffentlichen Rundfunks durch Steuern oder durch einen Beitrag zu erwarten waren, enthielten mehrere Vorschläge steuerbasierte Finanzierungsmodelle. Für die EU-Kommission bedeutete eine Finanzierung des Rundfunks durch Steuern keine Schwierigkeiten, wenn die Rundfunkanstalten ihre privatwirtschaftlichen und steuerfinanzierten Aktivitäten transparent trennen (Europäische Kommission 2006). Denkbar war etwa ein Modell, das die Kosten für den öffentlichen Rundfunk aus Gemeinschaftssteuern von Bund und Ländern deckt. Ein weiteres Modell sah vor, die Kosten aus bestehenden Landessteuern zu begleichen, ein drittes zog die Erhebung einer neuen Landessteuer in Erwägung. ${ }^{11}$

Trotz des scheinbaren Vorteils im Erhebungsverfahren schloss die MPK steuerbasierte Modelle aus unterschiedlichen Gründen aus. Ein wichtiger Grund bestand darin, dass eine Steuer grundsätzlich ohne eine Gegenleistung zu entrichten ist. Hinsichtlich der Finanzierung des Rundfunks hätte es mit dem Radio- und Fernsehangebot aber eine Gegenleistung gegeben. Weiterhin wären diese Steuereinnahmen wie alle anderen zweckfrei im Verfügungsbereich des Haushaltsgesetzgebers gewesen (Färber/Lücker 2011: 12). Dies hätte den Einfluss des Staates auf den öffentlich-rechtlichen Rundfunk erheblich erhöht und die Staatsferne der Rundfunkfinanzierung reduziert. Gleichzeitig wäre die Rundfunkfinanzierung den Anforderungen der Haushalte der Länder unterworfen gewesen, wie etwa der Schuldenbremse. Ein weiterer nicht unbedeutender Aspekt zulasten der steuerfinanzierten Finanzierungsvorschläge waren die zahlreichen Bestätigungen der Rundfunkgebühr durch das Bundesverfassungsgericht (BVerfG) in der Vergangenheit. Das

11 Für eine detaillierte Diskussion von steuerfinanzierten Vorschlägen siehe Kube 2013. 
BVerfG sah darin die Verwirklichung der Staatsferne des Rundfunks einschließlich seiner Gremien und seiner Finanzierung (Kube 2013: 30).

Die Erhebung des Rundfunkbeitrags durch die Finanzämter wurde ebenfalls in der Rundfunkkommission diskutiert. Doch diese Möglichkeit zeigte sich nicht mehrheitsfähig. Aus der Wissenschaft wurde das Argument geäußert, die Erhebung durch die Finanzämter sei ineffizienter als die Erhebung durch den Beitragsservice (Köster 2012). Weiterhin waren die Finanzämter wenig davon begeistert, eine weitere Aufgabe übertragen zu bekommen, weil sie nach eigener Auffassung durch Stellenkürzungen ohnehin überlastet waren. Schließlich bestand in den Rundfunkanstalten ein institutionelles Eigeninteresse, den Beitrag selbst einzuziehen.

\section{Von der inkrementellen Anpassung zur institutionellen Reform - oder von der Gebühr zum Beitrag}

Nach dem Ausschluss steuerbasierter Finanzierungsmodelle blieben zwei letzte Vorschläge in der engeren Auswahl der Ministerpräsidenten: eine vereinfachte Rundfunkgebühr und ein Rundfunkbeitrag. Die vereinfachte Rundfunkgebühr hätte die bestehenden Regeln graduell an die Problemlagen angepasst. In diesem Modell hätte man die Trennungen zwischen neuartiger und herkömmlicher sowie zwischen Grund- und Fernsehgebühr aufgehoben. Dann wäre nur noch eine Gebühr pro Wohn- bzw. Betriebsstätte angefallen. Das Modell hätte die mobilen Endgeräte (auch die Empfangsgeräte in KFZ) und die Endgeräte in Zweit- und Ferienwohnungen entweder zu einer Wohn- oder zu einer Betriebsstätte gerechnet. Die vereinfachte Rundfunkgebühr wäre damit eine inkrementelle Änderung gewesen, weil sich die Gebührenpflicht weiterhin an der Verfügbarkeit eines Empfangsgerätes orientiert hätte. Alternativ dazu beinhaltete ein geräteunabhängiges Modell den Vorschlag, die Finanzierung des öffentlichen Rundfunks an einen Haushalt bzw. an eine Betriebsstätte und eben nicht an Empfangsgeräte zu knüpfen - für die Rundfunkfinanzierung ein bis dahin gänzlich neues Konzept.

Ein neuer Rundfunkbeitrag bedeutete eine institutionelle Reform der bestehenden vertraglichen Regeln anstatt einer inkrementellen Anpassung. Beide Modelle hätten zur Lösung des Verteilungsproblems in der Rundfunkfinanzierung beigetragen. Allerdings hätten die Modelle unterschiedliche Konsequenzen gehabt. Die veränderte Rundfunkgebühr hätte zu größeren Einnahmeausfällen und damit zu einer höheren monatlichen Rundfunkgebühr geführt. Doch laut der Vorgabe der Ministerpräsidenten durfte der monatliche Betrag nicht weiter steigen. Dafür hätte sich die vereinfachte Rundfunkgebühr nahtlos in bestehende Regelungen und Praktiken eingefügt. Eine geräteunabhängige Abgabe stand vor der Schwierigkeit, neu in das bereits 
existierende Recht eingegliedert werden zu müssen. Die Verwerfungen zum bestehenden Recht waren demnach bei der vereinfachten Gebühr geringer als bei einem neu zu fassenden Beitrag. Demnach musste die rechtliche Zulässigkeit dieser Art von Abgabe juristisch geprüft werden. Gleichzeitig verlangte es nach einer neuen juristischen Definition von Haushalten und Betriebsstätten. ${ }^{12}$

Im Laufe des Jahres 2008 diskutierte die Ministerpräsidentenkonferenz die Machbarkeit und die Konsequenzen der vorgeschlagenen Modelle. Auf dem Treffen vom 22. bis 24. Oktober 2008 entschied die MPK, dass sie nur noch die Modelle der vereinfachten Rundfunkgebühr und einer geräteunabhängigen Abgabe konkretisieren wolle. Jedes der vorgeschlagenen Modelle enthielt stichhaltige Vor- und Nachteile, so dass das richtige Modell für die Zukunft der Rundfunkfinanzierung keineswegs auf der Hand lag. Eine institutionelle Reform war daher nicht zwingend und eine weitere inkrementelle Anpassung der Rundfunkfinanzierung möglich. Mithilfe der öffentlich-rechtlichen Rundfunkanstalten erarbeiteten die CdS und die Fachreferenten ein juristisches Gerüst aus Anknüpfungspunkten, Definitionen, Befreiungstatbeständen aus sozialen Gründen und Staffelungen für den nicht privaten Bereich. Entsprechend den Konkretisierungen rechneten die öffentlich-rechtlichen Rundfunkanstalten beide Modelle durch. Das Ziel: einen Gesamtbetrag von ca. 7,5 Milliarden $€$. Doch dies war kein einmaliger Vorgang, sondern ein iterativer Prozess. Öffentliche Verwaltungen, CdS, MPK, aber auch ARD und ZDF tauschten sich im Verlauf des Prozesses über die Auswirkungen von Änderungen auf den Gesamtertrag aus. Daran entzündete sich Kritik, weil die öffentlich-rechtlichen Rundfunkanstalten nicht nur Gegenstand der Beratungen, sondern selbst an den Verhandlungen beteiligt waren. Je nachdem, inwieweit Schätzungen den benötigten Gesamtbetrag von 7,5 Mrd. € verfehlten, veränderte die Rundfunkkommission die Befreiungen und Staffelungen.

Im Verlauf des Jahres 2009 fiel die Wahl auf den geräteunabhängigen Beitrag. Die Gründe dafür lagen darin, dass die öffentlich-rechtlichen Rundfunkanstalten bei der MPK und den CdS erhebliche Überzeugungsarbeit für dieses Beitragsmodell leisteten. Ausschlaggebend dürfte weiterhin gewesen sein, dass das Beitragsmodell keine modellbedingte Beitrags- bzw. Gebührensteigerung mit sich brachte und die Kontrolltätigkeit durch die beauftragten Dienste auf ein Minimum reduzierte. Im Juni 2010 stellte die MPK mithilfe der öffentlich-rechtlichen Rundfunkanstalten Eckpunkte einer ersten staatsvertraglichen Ausformulierung des neuen geräteunabhängigen Beitrags vor. Zuvor ließ sie jedoch im April 2010 ein Gutachten von

12 Dies verdeutlicht beispielsweise die Frage, ob Friedhöfe zu den Haushalten oder zu den Betriebsstätten zählen. 
Paul Kirchhof erstellen, das die juristischen Zweifel der politisch Verantwortlichen an der verfassungsrechtlichen Zulässigkeit beseitigte.

Im weiteren Verlauf ging es in den Verhandlungen zwischen den Ministerpräsidenten um punktuelle Anpassungen der staatsvertraglichen Ausformulierung. Trotz der Konkretisierungen des abstrakten Modells für aufkommende Einzelfälle folgten die beteiligten Akteure der grundsätzlichen Richtung. Die Mitarbeiterstaffel für die Beiträge im nicht privaten Bereich reduzierte die MPK für die beiden untersten Beitragsstaffeln, um vor allem Handwerksbetriebe zu entlasten. Auszubildende und Minijobber wurden darüber hinaus nicht in der Mitarbeiterstaffel mitgerechnet. Auch änderte die MPK die neue Formulierung dahingehend, dass nun Fahrzeuge auch auf Unternehmensstandorte angerechnet werden konnten. Für Hotel- und Gästehäuser beschloss sie eine Beitragsfreiheit der ersten Raumeinheit (Färber/Lücker 2011: 15). Damit wird das Hotel- und Gaststättengewerbe im Vergleich zur vorherigen Rundfunkgebühr entlastet. Gleiches gilt für Familien, weil bei ihnen die Mehrfachgebührenpflicht durch den Beitrag entfällt. Im Gegensatz dazu werden diejenigen mehr belastet, die bisher nur für alleinige Hörfunknutzung zahlten. Betriebsstätten zu gottesdienstlichen Zwecken stellte die MPK beitragsfrei. Blindtaube Personen müssen ebenfalls keinen Beitrag entrichten. Blinde oder taube Personen sind dagegen erst einmal beitragspflichtig. Zusätzlich erfolgte eine Änderung hinsichtlich der Kleingärten in den neuen Bundesländern. Diese wurden erst nach den vorgestellten Eckpunkten von der Beitragspflicht befreit.

\section{Institutionelle Reform trotz Politikverflechtung}

Die Umstellung der Rundfunkgebühr auf den Rundfunkbeitrag startete im Herbst 2006 und endete im Dezember 2011. Damit reagierten die Ministerpräsidenten für die Gebührenperiode 2013 bis 2016 auf den Problemdruck aufgrund des Verteilungs- und Niveauproblems. Der Zeitplan war durchaus ambitioniert. Auch wäre unter der Bedingung der technischen Konvergenz und der vielfältigen internetfähigen Endgeräte ohnehin kaum mehr ein Bürger mit plausibler Begründung ohne ein Tablet-Computer, Handy, Computer, Radio, Smartphone oder Fernsehen gewesen. Darüber hinaus verschwimmen schon jetzt die Grenzen zwischen den Endgeräten, so dass sich kaum glaubhaft darlegen ließe, ob sich nun ein Endgerät zur Nutzung von öffentlichem Rundfunk eignet oder nicht.

Nach der Modellentscheidung prüften die Ministerpräsidenten mit den öffentlichrechtlichen Rundfunkanstalten die Konkretisierung des Modells. Schließlich stellten die Ministerpräsidenten auch im Jahr 2010 ihre Eckpunkte für eine diesbezügliche Änderung des Rundfunkstaatsvertrages vor. Einzelne Aspekte wurden dann 
verändert und die Änderungen zum Rundfunkstaatsvertrag im Jahr 2011 durch die Landtage ratifiziert. Seit dem Januar 2013 gilt damit für jeden Haushalt und jede Betriebsstätte der Rundfunkbeitrag von 17,98 € pro Monat - unter der Berücksichtigung der entsprechenden Bestimmungen und Befreiungen.

Die Rundfunkkommission entschied sich schlussendlich gegen eine inkrementelle Anpassung und damit für die institutionelle Reform der Rundfunkfinanzierung. Dies ist überraschend, weil die Prognosen der Theorie der Politikverflechtung eine inkrementelle Anpassung eher vorhersagen als institutionelle Reformen (Scharpf 1985). Besonders bei Verteilungsproblemen erscheinen Reformen schwierig, weil dafür ein faktischer Konsens erforderlich ist. Mögliche Verlierer einer Reform vermögen es daher, Entscheidungen zu verhindern oder so zu verwässern, dass die Reform nicht mehr effektiv ist.

In horizontalen Verflechtungen ermöglichen Paketlösungen und Koppelgeschäfte eine Aufteilung des Kooperationsgewinns nach gerechten Verteilungsnormen (Zintel 1992). Doch in der Rundfunkfinanzierung zeigt sich für die Ministerpräsidenten kein Kooperationsgewinn, weil das Thema in der Öffentlichkeit negativ besetzt ist. Öffentlicher Druck sorgte demnach auch nicht für eine Verbesserung der Einigungsmöglichkeiten. Trotz des negativen Images der Rundfunkfinanzierung und ohne öffentlichen Druck gelang dennoch die institutionelle Reform.

\section{a) „Schleier des Nichtwissens“}

Die Rundfunkkommission stellte sich nicht der Debatte um gerechte Verteilungsnormen der Rundfunkfinanzierung. Stattdessen sollte der monatliche Betrag auch nach der institutionellen Reform stabil bleiben. Dies war schon zu Beginn der Verhandlungen eine Bedingung der MPK. Die Rundfunkkommission und die öffentlich-rechtlichen Rundfunkanstalten mussten daher Grundsätze für die Finanzierung diskutieren, ohne den späteren Ertrag sicher zu wissen. Zwar ermittelten die Rundfunkanstalten Prognosen auf Basis der diskutierten Änderungen, aber es war unklar, ob diese Prognosen zutreffen oder nicht. Gleiches gilt für die Befreiungen und Ausnahmebestimmungen, von denen ebenso unsicher war, in welchem Ausmaß diese in Anspruch genommen werden würden. Gegenstand der Verhandlungen war daher immer ein abstrakter Rundfunkbeitrag und kein konkreter Geldbetrag. Das Vertei- 
lungsproblem wurde lösbar, da alle Beteiligten an den Verhandlungen einem „Schleier des Nichtwissens“"13 unterlagen.

Um dennoch auf die Verteilung nach der institutionellen Reform Einfluss nehmen zu können, wurde im Verlauf der Ratifikation eine Evaluation des neuen Rundfunkbeitrags vereinbart. Von 2014 bis 2016 bewertet die KEF die Auswirkungen der institutionellen Reform auf der Grundlage der Erhebungsdaten des Jahres 2013. Die Erkenntnisse werden bei der Berechnung der Höhe des Rundfunkbeitrags für die Periode nach 2016 berücksichtigt, weil die erste Berechnungsperiode nach dem neuen Beitrag auch 2016 endet. Damit fällt die konkrete Verteilungsentscheidung in das KEF-Verfahren. Sie kann somit von den Grundsätzen des neuen Rundfunkbeitrags getrennt werden.

\section{b) Segmentierte Entscheidungsfindung}

Darüber hinaus ging es in den Verhandlungen ausschließlich um die Einnahmen in der Rundfunkfinanzierung. Mögliche Verfahren zu Kosten- bzw. Ausgabeneinsparrungen bei den öffentlich-rechtlichen Rundfunkanstalten waren nie auf der Tagesordnung der Rundfunkkommission. Die bereits existierenden Verfahren, wie das KEF-Verfahren, sollten unangetastet bleiben. Vorgeschlagene Finanzierungsmodelle, die das KEF-Verfahren tangierten, wurden daher von der Rundfunkkommission ausgeschlossen. Genauso wurden neue Maßnahmen zur Überprüfung von neuen Rundfunkinhalten, wie der Drei-Stufen-Test und der Gremienvorbehalt, nicht diskutiert.

Auch die Programmautonomie der Intendanten war in den Verhandlungen kein Thema, obwohl Programmentscheidungen vielfach mit einer Kostensteigerung verbunden sind. Das gleiche gilt beispielsweise für Entscheidungen über Moderatoren und deren Entlohnung. Für die institutionelle Reform der Rundfunkfinanzierung ist durch die Trennung von Grundsätzen sowie die Verteilung und die Reduktion der Entscheidungsalternativen eine segmentierte Entscheidungsfindung erkennbar (Scharpf/Reissert/Schnabel 1976: 60).

13 Der „Schleier des Nichtwissens“ stammt aus der Gerechtigkeitstheorie von John Rawls (1979). Rawls entwirft ein Gedankenspiel, in dem Menschen über eine zukünftige Gesellschaftsordnung entscheiden. Rawls argumentiert, dass die Menschen dann eine gerechte Entscheidung treffen, wenn sie nicht wissen, welchen Platz sie in der zukünftigen Gesellschaftsordnung einnehmen werden. Dieser „Schleier des Nichtwissens“ bezog sich zwar nicht wie im Konzept von Rawls auf die zukünftige Rolle der Entscheider, sondern auf die Finanzierung des Rundfunks. Trotzdem entfaltete sich die gleiche Wirkung mit Bick auf die Trennung von abstrakten Grundsätzen hin zu konkreten Verteilungen. 


\section{c) Positive und negative Koordination}

Entscheidend für die erreichte institutionelle Reform zeigte sich die Organisation des Verhandlungsprozesses. Der Rundfunkkommission standen die öffentlichrechtlichen Rundfunkanstalten gegenüber. Die Rundfunkanstalten verfügten über einen Informationsvorsprung gegenüber der Rundfunkkommission. Die KEF, als institutionelles Gegengewicht der Rundfunkanstalten, war an den Verhandlungen nicht beteiligt. Diesen unterschiedlichen Kapazitäten und Informationen folgte das Zusammenspiel von negativer und positiver Koordination. Die Rundfunkanstalten betrieben eine positive Koordination, indem sie einen Gesetzesvorschlag jenseits des Status quo erarbeiteten. Demgegenüber verfolgte die Rundfunkkommission eine negative Koordination. Es wurde von der Rundfunkkommission geprüft, welche negativen Auswirkungen der Gesetzesvorschlag für die Bundesländer haben würde. So erklären sich unter anderem die punktuellen Veränderungen des Rundfunkstaatsvertrages zwischen der Vorstellung der Eckpunkte und der Ratifikation des Staatsvertrages. Die spezifischen Anliegen konnten problemlos in das neue Konzept des Rundfunkbeitrags integriert werden. Dies gelang der Rundfunkkommission ohne eine grundsätzliche Änderung der institutionellen Reform.

Die förderliche Rolle eines durchdachten Zusammenwirkens von positiver und negativer Koordination wurde bereits in früheren Arbeiten herausgestellt (vlg. etwa Scharpf/Mohr 1994) und zeigte sich auch in den Verhandlungen zum Rundfunkbeitrag.

\section{Zusammenfassung und Ausblick}

Zwar erfolgte die Ratifikation durch die Landtage problemlos entsprechend dem anvisierten Zeitplan, doch sind damit nicht alle Unklarheiten bei der Konkretisierung des Rundfunkbeitrags ausgeräumt. Schon jetzt befassen sich zahlreiche Gerichte mit Klagen zum Rundfunkbeitrag. Zwischen den Jahren 2014 und 2016 befasst sich zudem eine Evaluierungsgruppe mit möglichen Änderungen für die Beitragsperiode nach 2016, um flexibel Feinjustierungen des Regelwerks durchführen zu können. Bezüglich der Finanzierungsunsicherheit lassen sich derzeit ebenfalls nur Tendenzen abschätzen; konkrete Beurteilungen sind dagegen (noch) nicht möglich. Bisherige Prognosen über die Entwicklung der Einnahmen der Rundfunkanstalten lassen vermuten, dass die Finanzierung durch den Beitrag auf eine sichere Grundlage gestellt wurde. Zwar besteht noch Unsicherheit in den Auswirkungen, etwa in den Befreiungstatbeständen auf den Gesamtertrag, aber zumindest für die Gebührenperiode 2013 bis 2016 konnte das Verteilungsproblem gelöst werden. 
Inwieweit sich die Akzeptanz des öffentlich-rechtlichen Rundfunks durch den neuen Rundfunkbeitrag erhöht, bleibt abzuwarten. Generell dürfte zunächst gelten, dass die Akzeptanz bei denjenigen, die durch den neuen Rundfunkbeitrag Vorteile erzielen, eher steigt. Das Niveau der Akzeptanz bei denjenigen, die durch den Beitrag dagegen stärker belastet werden, dürfte entsprechend sinken. Jenseits dieser Überlegungen ist der stabile Beitrag für die Zeit von 2013 bis 2016 sicherlich ein positives Zeichen für die Akzeptanz des öffentlich-rechtlichen Rundfunks. Auch eine zu erwartende geringere Prüftätigkeit der GEZ und der Auftragsdienste dürfte beim Beitragszahler auf ein positives Echo stoßen.

Doch diese Maßnahmen erscheinen nicht ausreichend, um das Akzeptanzniveau der öffentlich-rechtlichen Medien nachhaltig zu heben. Dem steht der Zwang zur grundsätzlichen Entrichtung des neuen Rundfunkbeitrags gegenüber. Sollten gerichtliche Auseinandersetzungen das Ergebnis erbringen, dass die Bürgerinnen und Bürger nicht für eine solche Zwangsabgabe verpflichtet werden können, gerät die Rundfunkfinanzierung erneut unter Problemdruck. In diesem Fall wäre nicht ausgeschlossen, dass die Öffentlichkeit die Grundsatzfrage thematisiert, ob die jährlichen Kosten von 7,5 Mrd. $€$ für den öffentlich-rechtlichen Rundfunk überhaupt gerechtfertigt sind.

\section{Literatur}

Benz, Arthur/Scharpf, Fritz W./Zintl, Reinhard, 1992: Horizontale Politikverflechtung. Zur Theorie von Verhandlungssystemen, Frankfurt a. M.

Degenhart, Christoph, 2011: Verfassungsrechtliche Zweifelsfragen des Rundfunkbeitragsstaatsvertrags, in: Zeitschrift für Urheber- und Medienrecht 55 (3), 193-200.

Europäische Kommission, 2006: Staatliche Beihilfen: Öffentlich-rechtliche Rundfunkanstalten in Deutschland - Presseerklärung von EU-Wettbewerbskommissarin Neelie Kroes und den Ministerpräsidenten Kurt Beck und Edmund Stoiber, MEMO/06/273, http://europa.eu/rapid/press-release_MEMO-06-273_de.htm? locale $=$ en (Stand: 15.11.2013).

Falkner, Gerda, 2010: The EUs Decision Traps: Comparing Policies, Oxford.

Färber, Hans W./Lücker, Matthias, 2011: Die Ersetzung der Rundfunkgebühr durch einen Rundfunkbeitrag, Arbeitspapiere des Instituts für Rundfunkökonomie Köln, Heft 285.

Große-Hüttmann, Martin, 2004: „Wir müssen aus dem Mischmasch raus”: Die Europafähigkeit des deutschen Föderalismus, in: Frank Decker (Hrsg.), Föderalis- 
mus an der Wegscheide? Optionen und Perspektiven einer Reform der bundesstaatlichen Ordnung, Wiesbaden, 203-222.

Hanfeld, Michael, 2013: Jetzt rollt die Klagewelle an, FAZ, 11.1.2013, http:// www.faz.net/aktuell/feuilleton/medien/neuer-rundfunkbeitrag-jetzt-rollt-dieklagewelle-an-12020705.html (Stand: 22.8.2013).

Hauser, Jan, 2013: Zu viel GEZahlt: Sixt klagt gegen Rundfunkabgabe, FAZ, 20.8.2013, http://blogs.faz.net/medienwirtschaft/2013/08/20/zu-viel-gezahltsixt-klagt-gegen-rundfunkabgabe-279/ (Stand: 22.8.2013).

Heinz, Dominic, 2012: Varianten von Politikverflechtung: Der Rundfunkbeitragsstaatsvertrag, in: Europäisches Zentrum für Föderalismus-Forschung Tübingen (Hrsg.), Jahrbuch des Föderalismus 2012, Baden-Baden, 169-178.

Hicketier, Knut, 1998: Geschichte des deutschen Fernsehens, Stuttgart.

Hoff, Dieter, 2001: Technische Konvergenz - Fakten und Perspektiven, Arbeitspapiere des Instituts für Rundfunkökonomie Köln, Heft 147.

Hrbek, Rudolf, 1986: Doppelte Politikverflechtung: deutscher Föderalismus und europäische Integration. Die deutschen Länder im EG-Entscheidungsprozess, in: Deutsch-Französisches Institut (Hrsg.), Die deutschen Länder und die europäische Gemeinschaft, Ludwigsburg, 17-36.

Jeffery, Charlie, 2008: Groundhog Day: The Non-Reform of German Federalism, Again, in: German Politics 17 (4), 587-592.

Kirchhof, Paul, 2010: Gutachten über die Finanzierung des öffentlich-rechtlichen Rundfunks, Heidelberg.

Knothe, Matthias, 2010: (Rundfunk-)Staatsverträge - Faktische Gesetzgebung der Regierung unter Ausschluss der Parlamente?, in: Zeitschrift für Rechtspolitik (6), 181-184.

Kops, Manfred, 2009: Das deutsche duale Rundfunksystem - Entstehungsgeschichte, derzeitige Ausgestaltung und absehbare Veränderungen, Arbeitspapiere des Instituts für Rundfunkökonomie Köln, Heft 250.

Köster, Julia, 2012: Einzug von Rundfunkbeiträgen durch die Finanzämter - eine tragfähige Alternative?, in: Zeitschrift für Urheber- und Medienrecht 56 (12), 946-953.

Krick, Eva/Blumenthal, Julia von, 2013: Regieren in der Politikverflechtung: Parteienwettbewerb und Verhandeln, in: Karl Rudolf Korte/Timo Grunden (Hrsg.), Handbuch Regierungsforschung, Wiesbaden, 287-296.

Kropp, Sabine, 2010: Kooperativer Föderalismus und Politikverflechtung, Wiesbaden.

Kube, Hanno, 2013: Der Rundfunkbeitrag - Rundfunk- und finanzverfassungsrechtliche Einordnung. Rechtsgutachten, Mainz. 
Meulemann, Heiner, 2009: Verdrängt das Internet die Massenmedien? Medienpräferenzen und die Individualisierung der Mediennutzung, Arbeitspapiere des Instituts für Rundfunkökonomie Köln, Heft 249.

Moore, Carolyn/Eppler, Annegret, 2008: Disentangling Double Politikverflechtung? The Implications of the Federal Reforms for Bund-Länder Relations in Europe, in: German Politics 17 (4), 488-508.

Nägele, Frank, 1996: Regionale Wirtschaftspolitik im kooperativen Bundesstaat. Ein Politikfeld im Prozess der deutschen Wiedervereinigung, Baden-Baden.

Oates, Wallace E., 1972: Fiscal federalism, New York.

Ostrom, Vincent, 1971: The political theory of a compound republic: a reconstruction of the logical foundations of American democracy as presented in The Federalist, Blacksburg.

Rawls, John, 1979: Eine Theorie der Gerechtigkeit, Frankfurt a. M.

Reiter, Ulrich, 2010: Textdokumentation zur Veröffentlichung im Internet über die öffentliche Anhörung in der 54. Sitzung des Ausschusses für Bundes- und Europaangelegenheiten sowie Medien am 26.11.2010. Adrs. EUR/5/132, Magdeburg.

Renzsch, Wolfgang, 1991: Finanzverfassung und Finanzausgleich. Die Auseinandersetzungen um ihre politische Gestaltung in der Bundesrepublik Deutschland zwischen Währungsreform und deutscher Vereinigung (1948 bis 1990), Bonn.

Sarcinelli, Ulrich, 2006: Medienpolitik: Meinungsvielfalt, Demokratie und Markt, in: Christoph Scholz (Hrsg.), Handbuch Medienmanagement, Wiesbaden, 195-219.

Schächter, Markus, 2010: Textdokumentation zur Veröffentlichung im Internet über die öffentliche Anhörung in der 54. Sitzung des Ausschusses für Bundesund Europaangelegenheiten sowie Medien am 26.11.2010. Adrs. EUR/5/132, Magdeburg.

Scharpf, Fritz W., 1978: Die Theorie der Politikverflechtung: ein kurzgefaßter Leitfaden, in: Joachim J. Hesse (Hrsg.), Politikverflechtung im föderativen Staat, Baden-Baden, 21-31.

Scharpf, Fritz W., 1985: Die Politikverflechtungs-Falle: Europäische Integration und deutscher Föderalismus im Vergleich, in: Politische Vierteljahresschrift 24 (4), 323-356.

Scharpf, Fritz $W$., 1992: Koordination durch Verhandlungssysteme: Analytische Konzepte und institutionelle Lösungen, in: Arthur Benz/Fritz W. Scharpf/Reinhard Zintl (Hrsg.), Horizontale Politikverflechtung. Zur Theorie von Verhandlungssystemen, Frankfurt, 51-96. 
Scharpf, Fritz W., 2006: The Joint-Decision-Trap Revisited, in: Journal for Common Market Studies 44 (4), 845-864.

Scharpf, Fritz W./Mohr, Matthias, 1994: Efficient Self-Coordination in Policy Networks. A Simulation Study, MPIFG Discussion Paper Köln, 94/1.

Scharpf, Fritz W./Reissert, Bernd/Schnabel, Fritz, 1976: Politikverflechtung: Theorie und Empirie des kooperativen Föderalismus in der Bundesrepublik, Kronberg.

Scheller, Henrik, 2008: Politikverflechtung als Funktionsvoraussetzung des Bundesstaates? Zum Umgang mit dem Faktischen in der deutschen Föderalismusdiskussion, in: der moderne staat 1 (2), 469-483.

Scheller, Henrik/Schmid, Josef (Hrsg.), 2008: Föderale Politikgestaltung im deutschen Bundesstaat. Variable Verflechtungsmuster in Politikfeldern, Baden-Baden.

Schönenborn, Jörg, 2012: Stellungnahme: Ein Beitrag zur Funktionsfähigkeit der Gesellschaft, 27.12.2012, http://www.ard.de/intern/rundfunkbeitrag-kommen tar-schoenenborn $/-/ \mathrm{id}=1886 / \mathrm{nid}=1886 / \mathrm{did}=2773222 / 1 \mathrm{kbcfnk} / \mathrm{index} \cdot \mathrm{html}$ (Stand: 22.8.2013).

Wachendorfer-Schmidt, Ute, 2003: Politikverflechtung im vereinigten Deutschland, Wiesbaden.

Zintl, Reinhard, 1992: Kooperation und Aufteilung des Kooperationsgewinns bei horizontaler Politikverflechtung, in: Arthur Benz/Fritz W. Scharpf/Reinhard Zintl (Hrsg.), Horizontale Politikverflechtung. Zur Theorie von Verhandlungssystemen, Frankfurt a. M., 97-146.

Korrespondenzanschrift:

Dr. Dominic Heinz

Technische Universität Darmstadt

Institut für Politikwissenschaft

Residenzschloß

64283 Darmstadt

E-Mail: heinz@pg.tu-darmstadt.de 